\title{
Histological, histomorphometric and microtomographic analyses of retrieval hip resurfacing arthroplasty failed at different times
}

\author{
Francesca Salamanna ${ }^{1 *}$, Milena Fini ${ }^{1,2}$, Annapaola Parrilli ${ }^{1}$, Matteo Cadossi ${ }^{3}$, Nicolò Nicoli Aldini ${ }^{1,2}$, \\ Gianluca Giavaresi ${ }^{1,2}$, Deianira Luciani ${ }^{3}$ and Sandro Giannini ${ }^{3}$
}

\begin{abstract}
Background: Metal-on-metal hip resurfacing arthroplasty (HR) has been gaining popularity especially for young and active patients. Although different series report good mid-term results, the long-term outcome and failure mechanisms are still concerning. In this consecutive revision case series, 9 retrieved specimens of a failed Birmingham Hip Resurfacing (BHR) were divided according to the time to fracture: 3 specimens failed at less than 6 months (Group 1), 3 failed between 6 months and 3 years (Group 2) and 3 failed later than 3 years (Group 3). The objective of the study was to examine by a specific quantitative histomorphometry and microtomography (micro-CT) method the characteristics of bone quality and its microarchitecture in retrieved metal-on-metal HR.

Methods: A series of 948 BHR were performed between 2001 and 2009. Among these implants 10 failures occurred and nine of these underwent revision surgery and were examined by histomorphometry and micro-CT.

Results: Histomorphometry showed a significant increase in trabecular separation (Tb.Sp) in Group 3 in comparison with Group 1 (113\%, $\mathrm{p}<0.05)$. In the top region, micro-CT showed that Groups 2 and 3 presented significant lower bone volume (Group 2: 61\%, $p<0.005$; Group 3: 1\%, p < 0.05), trabecular number (Group 2: 53\%, $p<0.005$; Group 3: 40\%, p < 0.05), and higher Tb.Sp (Group: 71\%,p<0.05) when compared to Group 1.

Additionally, histomorphometry showed that the top regions in Group 1 had a significantly lower mean percentage of empty osteocyte lacunae than the top regions in both Group 2 and $3(p<0.05)$.

Conclusions: This study showed that the morphometric parameters considered are crucial for a good understanding of mechanical properties of HR and may be of significant importance in the pathogenesis of HR failure particularly in the development of late fractures.
\end{abstract}

Keywords: Hip resurfacing arthroplasty, Failure, Histomorphometry, Microtomography

\section{Background}

Joint replacement is continuously evolving to reduce the invasiveness of surgery, prolong the implant life, decrease complications and improve the patient's life quality. Resurfacing hip arthroplasty is emerging as an alternative to conventional total hip arthroplasty and has been proposed as an option for the treatment of degenerative hip disease in young, active individuals [1]. HR

\footnotetext{
* Correspondence: francesca.salamanna@ior.it

'Laboratory of Biocompatibility, Innovative Technologies and Advanced Therapies, Rizzoli Research Innovation Technology, Rizzoli Orthopaedic Institute, Via di Barbiano 1/10, 40136, Bologna, Italy

Full list of author information is available at the end of the article
}

may present benefits over total hip replacement because femoral bone stock is maintained, there is reduced wear compared with high density polyethylene, it has a large femoral head that could be reduced dislocation rate, it is said to offer the patient increased levels of postoperative activity and is easy to convert into a stemmed prosthesis [2]. The general opinion about this procedure is mainly divided into a favorable one advocated by McMinn et al. [3] and a negative one supported by Spierings et al. [4]. The mid-term results of Birmingham Hip Resurfacing (BHR) suggest a survival rate of about $98 \%$ at five years. However, Spierings et al. still consider resurfacing as an experimental design for investigational use only, until

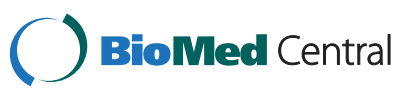


long-term follow up confirms its superiority in comparison with total hip replacement $[3,4]$. Recently various complications, such as femoral neck fracture [5-7], avascular necrosis $[8,9]$ and pseudotumour formation $[10,11]$, as well as unexplained pain, aseptic loosening [12], and osteolysis [4] have been reported. To improve the technique and the success of the treatment, experimental preclinical models can be used to allow the evaluation of biomechanics, biocompatibility, bioactivity and biofunctionality on innovative biomaterials, prosthetic devices and combined therapies. Nevertheless, the retrieval of failed prostheses and the analysis of human implanted devices is one of the most valuable tools to provide information about prostheses that have been submitted to clinical loading and biological and chemical micro-environment during their stay in the body [13]. Indeed, analysis of retrievals can show the histopathological response and the mechanisms of failure [14]. Currently, radiology and histology are the most common procedures to study failed bone implants and some authors have used these techniques to evaluate bone necrosis and fracture risk [6,10,15-18]. Although it is clear that we require a better understanding of the failure mechanisms of the current generation of metal-on metal HR implants, no studies have ever used histomorphometric and microtomographic evaluation to evaluate the characteristics of bone quality and its microarchitecture in retrieved metal-on-metal HR. In fact, from a literature search of the entire MEDLINE database (PubMed research engine) using the MeSH database terms ("hip arthroplasty" [Mesh] OR "hip resurfacing" [Mesh]) AND ("histomorphometric evaluations" [Mesh] OR " $x$ ray microtomography" [Mesh]) no studies were found. Histomorphometry provides information regarding bone tissue and cell dynamics. Similarly, a microtomographic evaluation of the bone structure gives a real estimate of its morphology, especially when it is carried out directly on the entire volume without using predefined volumetric models (plate or rod-model) [19]. Moreover, good correlations were found between the structural parameters determined by microtomography $(\mu \mathrm{CT})$ images and those assessed on histomorphological slices [20].

Therefore, the main goal of this study was to analyze and examine the characteristics of bone quality and its microarchitecture in retrieved metal-on-metal BHR by a new and specific quantitative histomorphometry and $\mu \mathrm{CT}$ method, never used before. This novel and innovative technique was performed to evaluate whether these $2 \mathrm{D}$ and $3 \mathrm{D}$ quantitative measurements might be applied to this field of research and give further insight into the failure mechanisms of these implants. This methodology was applied to a small consecutive revision case series taking into account different times to fracture and bone areas located at different distances from the HR dome.

\section{Methods}

\section{Patient cohort}

This is a retrospective observational study in which the protocol was explained to the patients and they gave written informed consent before entering the study (Determination of 20 March 2008, Italian Medicines Agency - AIFA).

A series of 948 (373 female and 575 male) HR (Birmingham Hip Resurfacing, Midland Medical Technologies Ltd, Birmingham, UK: now Smith\&Nephew) was performed in our ward at Rizzoli Orthopaedic Institute between early 2001 and late 2009 of whom 941 patients were available for follow-up. Among these implants 10 fractures occurred. Nine of these fractures underwent revision surgery at the Rizzoli Institute and they constitute the series of this study. All these patients underwent HR for primary arthritis of the hip through a posterolateral approach. The patients' characteristics of failed HR are reported in Table 1.

Fractures were divided into three groups:

- Gross fractures that occurred soon after surgery, earlier than 6 months (Group 1) which presented a pattern involving the implant rim. These fractures were

Table 1 Summary of the cases: patients gender and age (at the time of the primary operation) implant sizes, operation site, time to revision (F: female; M: male)

\begin{tabular}{|c|c|c|c|c|c|c|c|}
\hline Group & Time to Revision & Age/Gender & Implant & Notching & Cup inclination & Stem Neck angle & Side \\
\hline \multirow[t]{3}{*}{ Group 1} & 3 weeks & $70 / \mathrm{M}$ & 46- mm head -52-mm cup & Present & $54^{\circ}$ & $7^{\circ}$ & Left \\
\hline & 2 month & $60 / F$ & 46-mm head, 52-mm cup & Present & $52^{\circ}$ & $0^{\circ}$ & Right \\
\hline & 5 month & $50 / F$ & 44- mm head -50-mm cup & Present & $47^{\circ}$ & $6^{\circ}$ & Right \\
\hline \multirow[t]{3}{*}{ Group 2} & 14 month & $69 / F$ & 42- mm head -52-mm cup & Absent & $45^{\circ}$ & $0^{\circ}$ & Right \\
\hline & 36 month & $47 / F$ & 46- mm head -52-mm cup & Absent & $46^{\circ}$ & $0^{\circ}$ & Right \\
\hline & 36 month & $44 / F$ & 42-mm head-48-mm cup & Absent & $67^{\circ}$ & $1^{\circ}$ & Right \\
\hline \multirow[t]{3}{*}{ Group 3} & 4 years & 53/M & 50- mm head -56-mm cup & Absent & $52^{\circ}$ & $3^{\circ}$ & Left \\
\hline & 7 years & $50 / F$ & 42- mm head-48-mm cup & Absent & $60^{\circ}$ & $4^{\circ}$ & Left \\
\hline & 8 years & $51 / F$ & 46- mm head -52-mm cup & Absent & $54^{\circ}$ & $10^{\circ}$ & Left \\
\hline
\end{tabular}


characterized by diffuse reactive changes and varying degrees of perfusion of the proximal bone depending on the vascular injury. It was hypothesized that these aspects may be related to the surgical technique leading to biomechanical changes in the femoral neck by the notch (acute biomechanical fractures);

- Fractures that occurred between 6 months and 3 years (Group 2) and fractures that occurred later than 3 years (Group 3) were defined as late fractures, completely inside the femoral head, with extensive evidence of osteonecrosis. These two groups were divided arbitrarily to highlight the possible presence of a phenomenon that progresses with time. Macroscopically, in each group, necrotic bone tissue appeared pale and white-yellowish with scattered calcifications. One patient of Group 2 experienced a pseudotumor of ileopsoas with an aseptic lymphocytic vasculitis-associated lesions (ALVAL) at 3 years follow up. The acetabular inclination angle was $67^{\circ}$, thus suggesting the presence of edge wear. Metallic debris was evident macroscopically. All patients of Group 3 presented evident macroscopic signs of metallosis without soft tissue involvement, suggesting that osteonecrosis might be also developed by metal corrosion phenomena.

\section{Surgery}

With the patient well secured in the lateral position and under general or spinal anesthesia an extended posterior approach to the hip joint was used in a clean-air operating theatre. The short external rotators were released, the gluteus maximus was detached from its insertion at the linea aspera, and a circumferential capsulotomy was performed. The femoral head was dislocated anteriorly and the acetabulum reamed sequentially. Peripheral acetabular osteophytes were excised and a trial component which was $1 \mathrm{~mm}$ smaller than the intended final implant was used to confirm that a tight fit had been obtained. If this fixation was satisfactory, the definitive acetabular component was then impacted. Standard instrumentation was used to align and position the guide rod for the preparation of the femoral head using the lateral cortical pin and out-rigger. The head was reamed to house a femoral component that matched the implanted acetabular component. The femoral implant was positioned and secured with Simplex (Howmedica International, Limerick, Ireland) low viscosity cement. The hip was then reduced and the short external rotators and gluteus maximus tendon repaired.

\section{Histological and histomorphometric analyses}

At revision, the femoral component together with the femoral head and neck bone was resected en bloc and immediately placed in buffered $4 \%$ paraformaldehyde. No acetabular components were removed. After fixation, the bone-metal composite specimens were dehydrated by placing them in graded series of increasing percentage of alcohol with one step in 50\% alcohol, one step in $75 \%$ alcohol, two steps in $95 \%$ alcohol, and two steps in $100 \%$ alcohol, for 48 hrs per each step. After dehydration, the undecalcified specimens were infiltrated by exposing them to methyl methacrylate solution (Merck, Germany). The infiltration was completed with use of methyl methacrylate combined with benzoyl peroxide (Sigma-Aldrich) and poly-methyl-methacrylate (PMMA) (Sigma-Aldrich) under vacuum condition for four days. The different steps of the study are summarized in Figure 1. The embedded specimens were cut in middle along the coronal plane by a saw with a diamond-coated band (EXAKT, GmbH \& Co., Norderstedt, Germany). The orientation of the specimens during the cutting process was preserved, thus maintaining a low feed force coupled with the automatic control of the cutting band. One section of about $0.5 \mathrm{~cm}$ thick and two sections of $350 \pm 100 \mu \mathrm{m}$ thick were obtained for each HR sample. The implanted device was removed from the first section and the section was analyzed by $\mu \mathrm{CT}$ as described below. Then, it was automatically thinned (EXAKT Systems) to $15 \pm 5 \mu \mathrm{m}$ with different abrasive papers (EXAKT Abrasive Disc), from 80 to 2000 grit in steps of 15 minutes each and used for histomorphometric measurements. By the same procedure the other two sections of $\mathrm{HR}$ retrieved were automatically thinned to $60 \pm 10 \mu \mathrm{m}$ and used to measure the contact between bone and prosthetic stem. Next, the sections were stained with Toluidine Blue, Acid Fuchsin, Fast Green, and processed for routine histological analyses. Histological analyses were performed by using a transmission and polarized light AxioSkop Microscope (Carl Zeiss $\mathrm{GmbH}$, Germany) at a magnification from $1.25 \mathrm{x}$ to $20 \mathrm{x}$. After prosthesis removal, two different compartments of the femoral head were considered: A lateral and B medial. Each compartment was split into 3 regions of interest (ROI) depending on the distance from the HR dome: within $0.8 \mathrm{~cm}$ (top), from 0.8 to $1.6 \mathrm{~cm}$ (central) and from 1.6 to $2.4 \mathrm{~cm}$ (bottom). Finally, histomorphometric analyses were carried out with computerized image analysis Axio-Vision-4.6 (Carl Zeiss). Bone histomorphometry measurements were taken semi-automatically at a magnification of $1.25 \mathrm{x}$ by two experienced blinded investigators, by dividing the sections of compartments $\mathrm{A}$ and $B$ into different quadrants. The bone-to-implant-contact (BIC) was measured at the interface between bone and prosthesis stem as the rate between the stem surface directly in contact with bone without the interposition of fibrous tissue/the total interface length $x 100$ (\%). The bone histomorphometric parameters were measured in accordance with the Histomorphometry Nomenclature by the Committee of the American Society for Bone and Mineral Research [21]: 


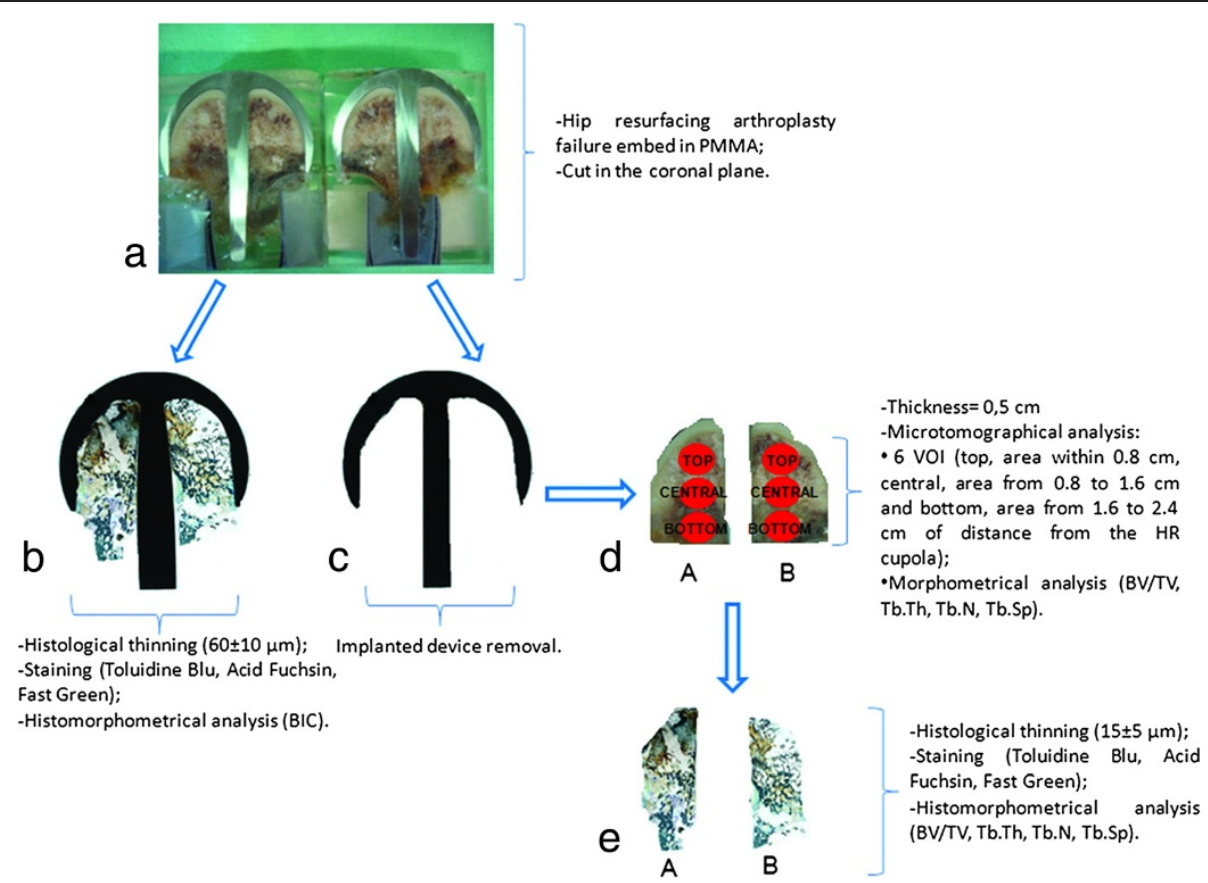

Figure 1 Schematic representation of the methodology for sample analyses. Epson 2480 Scanner, 600dpi of resolution. a) The specimens were embedded in PMMA and cut along the coronal plane, b) sections containing the implants were used for histology and histomorphometric measurement $(\mathrm{BIC}), \mathbf{c}$ ) after the removal of the prosthesis, $\mathbf{d}$ ) two bone compartments ( $\mathrm{A}$ and $\mathrm{B}$ ) were used for $\mu \mathrm{CT}$, e) thinned and processed for routine histological and histomorphometric analyses.

- Bone Volume (BV/TV,\%): the whole spongy bone area, expressed as a percentage of the total tissue area in the sampling site and converted to a volume;

- Trabecular Number (Tb.N, $\mathrm{mm}^{-1}$ ): index of density of trabeculae;

- Trabecular Thickness (Tb.Th, $\mu \mathrm{m})$ : index of the width of trabeculae;

- Trabecular Separation (Tb.Sp, $\mu \mathrm{m}$ ): index of the distance of trabeculae;

- Cement Thickness (Cm.Th): index of the width of the cement on the dome surface.

For each sample, the mean percentage of empty lacunae in five regions of interest in the top, central and bottom part, at a magnification of $20 x$, was determined by two experienced blinded investigators using the method of Steffen et al. [22].

\section{Microtomographic analysis}

As shown in Figure 1, $\mu \mathrm{CT}$ assessment was carried out on a 0.5 -cm-thick embedded section of the samples using the Skyscan 1172 computed microtomographic system (Kontich, Belgium). The scans were performed with a $100 \mathrm{kV}$ voltage source and $100 \mu \mathrm{A}$ current source. Images were acquired with a pixel size of $12 \mu \mathrm{m}$, an aluminum filter $0.5 \mathrm{~mm}$, and a sample rotation step of $180^{\circ}$ and $0.4^{\circ}$. The scans were later reconstructed to obtain microtomographic sections. Reconstruction was performed by using NRecon (v1.6.2.0) software: a specific alignment was used for each sample and a medium intensity ring artifact correction was applied. Microtomographic 3D analyses were performed using CTAn (v.1.10.1.3) software and, as for histologic evaluation, considering 3 Volumes of interest (VOI) in each compartment ( $\mathrm{A}$ and $\mathrm{B}$ ): at the top, within $0.8 \mathrm{~cm}$ from the HR dome, in the centre, from 0.8 to $1.6 \mathrm{~cm}$ from the HR dome, and at the bottom, 1.6 to $2.4 \mathrm{~cm}$ from the HR dome. The morphometric parameters considered were derived in part from those already defined by Parfitt [21]:

- Bone volume density (BV/TV,\%), expressed as a ratio between the volume of bone measured in the VOI and the total volume of the considered VOI;

- Trabecular thickness (TbTh, $\mu \mathrm{m}$ ) measured as a true model-independent 3D value;

- Trabecular separation ( $\mathrm{TbSp}, \mu \mathrm{m})$, derived from the volume-based local thickness just applying the method to the space between trabeculae;

- Trabecular number $\left(\mathrm{TbN}, \mathrm{mm}^{-1}\right)$, defined as: $1 /(T b T h+T b S p)$.

\section{Statistical analysis}

Statistical analysis was performed by using the SPSS Inc v.12 software. Data were reported as Mean \pm SD at a significant level of $\mathrm{p}<0.05$. After checking normal distribution 
(Shapiro-Wilks test) and homogeneity of variance (Levene test), the non-parametric Kruskall-Wallis test followed by Mann-Whitney test with Monte Carlo methods to compute probability were carried out to compare histomorphometric results among groups.

\section{Results \\ Histological results \\ Group 1}

The mean time-to-revision was $10.3 \pm 8.7$ weeks. No difference was found by histological analysis in this group (Figure 2). A thin layer of cement was present at the bone-dome interface and intraosseous cement penetration was recognized by its hard consistency, fine granular structure, and color in the top regions. Focal areas of osteonecrosis with trabecular lamellar bone with empty lacunae were observed in the central and bottom regions (Figure 2a,b). In the bone areas adjacent to the HR dome (top ROI) normal bone microarchitecture and morphology with osteocyte nuclei in the lacunae were observed (Figure 2a). In the 5-month case the areas of focal osteonecrosis with bone trabeculae without stainable osteocytes were associated with signs of appositional new bone formation.

\section{Group 2}

The mean time-to-revision was $28.6 \pm 12.7$ months. No differences were observed among the 3 analyzed prostheses that had failed from 14 to 36 months (Figure 2c,d). A cement mantle was present at the dome and intraosseous cement penetration was observed in the top region and in a small part of the central region. An absence of osteocyte nuclei within bone lacunae was observed in all cases in all ROIs. Thickened cancellous bone trabeculae were sometimes observed with extensive formation of appositional new bone on the surface of necrotic trabeculae (Figure 2c). Signs of metallosis with infiltration and accumulation of metallic wear debris inside the periprosthetic structures were clearly visible in the two patients that failed at longer follow up times (Figure 2d). At 36 months a considerable amount of connective tissue was observed.

\section{Group 3}

The mean time-to-revision was $6.3 \pm 2.1$ years. Sectioning of the implant revealed a thin layer of cement at the dome and a penetration of cement deep into the bone was observed in the top and central region. Femoral head section analyses showed a decrease in bone mass with partial necrosis in each examined sample. Histological examination confirmed the presence of a massive metallosis revealing granulomatous tissue with extensive pigmented deposits in all examined cases, which was more evident at 7 and 8 years with bone rarefaction present in all ROIs of the femoral head (Figure 2e,f).

\section{Histomorphometric results}

The 2D histomorphometric tests were performed on the sections where the implanted device was present for BIC and $\mathrm{Cm}$. Th measurement and in those where the HR had been removed for BV/TV, Tb.Th, Tb.N, Tb.Sp and percentage of empty lacunae measurements. The results of histomorphometric parameters are reported in Figure 3a and b. The Kruskal-Wallis
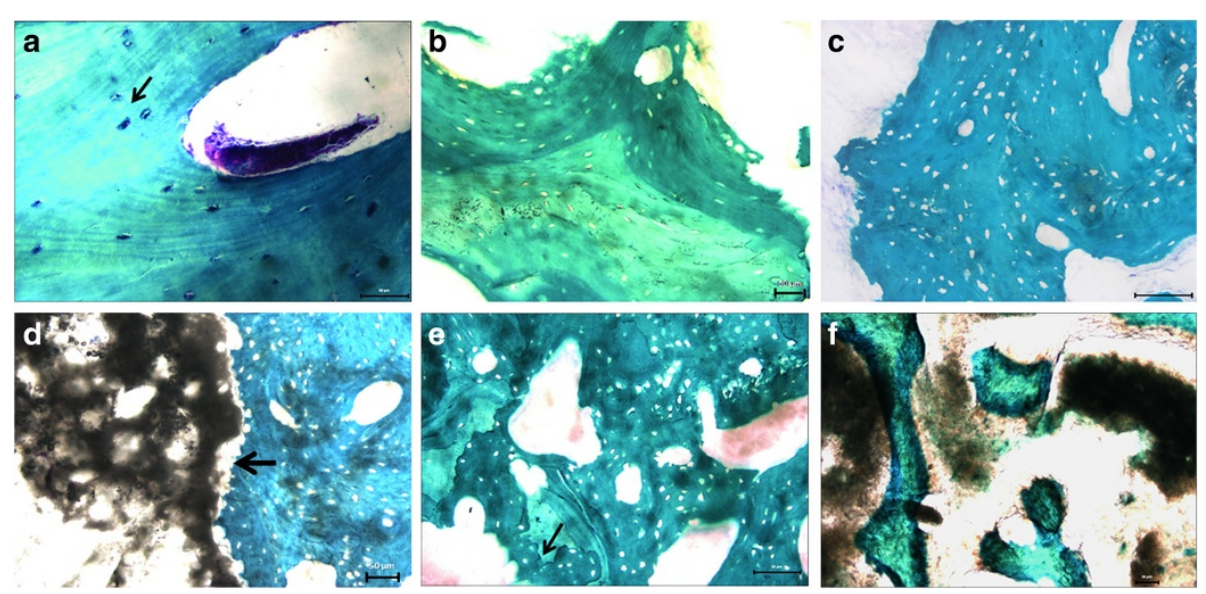

Figure 2 Histology of specimens revised in Group $1(a, b)$, Group $2(c, d)$ and Group $3(e, f)$; sections are representative of bone tissue at increasing distances from the HR dome: within $0.8 \mathrm{~cm}$ (top) (a, c, e), from 1.6 to $2.4 \mathrm{~cm}$ (b, d, f) (bottom). Toluidine Blue, Acid Fuchsin, Fast Green staining. a) trabecular lamellar bone with evident evenly-spread osteocytes, orientated with the longest axis in the direction of the lamellae contained in the bone lacunae (arrows), resolution 20x; b) necrotic bone tissue, resolution 10x; c) necrotic tissue, resolution 20x; d) necrotic bone tissue infiltrated with aggregates of small dark metal wear-debris particles (metallosis) (arrows), resolution 10x; e) loss of normal trabecular bone microarchitecture, uneven edges due to the resorption of necrotic bone (arrows) resolution 20x; f) metallosis in close association with the necrotic bone trabeculae, resolution 10x. 
test highlighted statistically significant differences between groups for BIC $(p=0.004)$. The Mann-Whitney test showed that Group 3 presented statistically significantly lower BIC values $(71 \%, p=0.004)$ when compared to those of Group 1. The results of the percentage of empty lacunae obtained from each separate ROI (top, middle and bottom) are summarized in Figure 3b. Statistically significant differences were found for empty lacunae in Group $1(p=0.05)$ between the top region and the others. Additionally, the top regions in Group 1 had a significantly lower mean percentage of empty osteocyte lacunae than top regions in both Group 2 and Group $3(p=0.05)$.

\section{Microtomographic results}

The 3D microtomographic analysis was carried out on the sections after the removal of the implanted device. The results of $\mu \mathrm{CT}$ parameters for each patient are reported in Figure 4. Data are in parallel with those of histomorphometric results and showed that bone rarefaction (as measured by BV/TV, Tb.N, Tb.Sp) progressively changes over time (Figure 5a,b). The comparison between Group 1 and
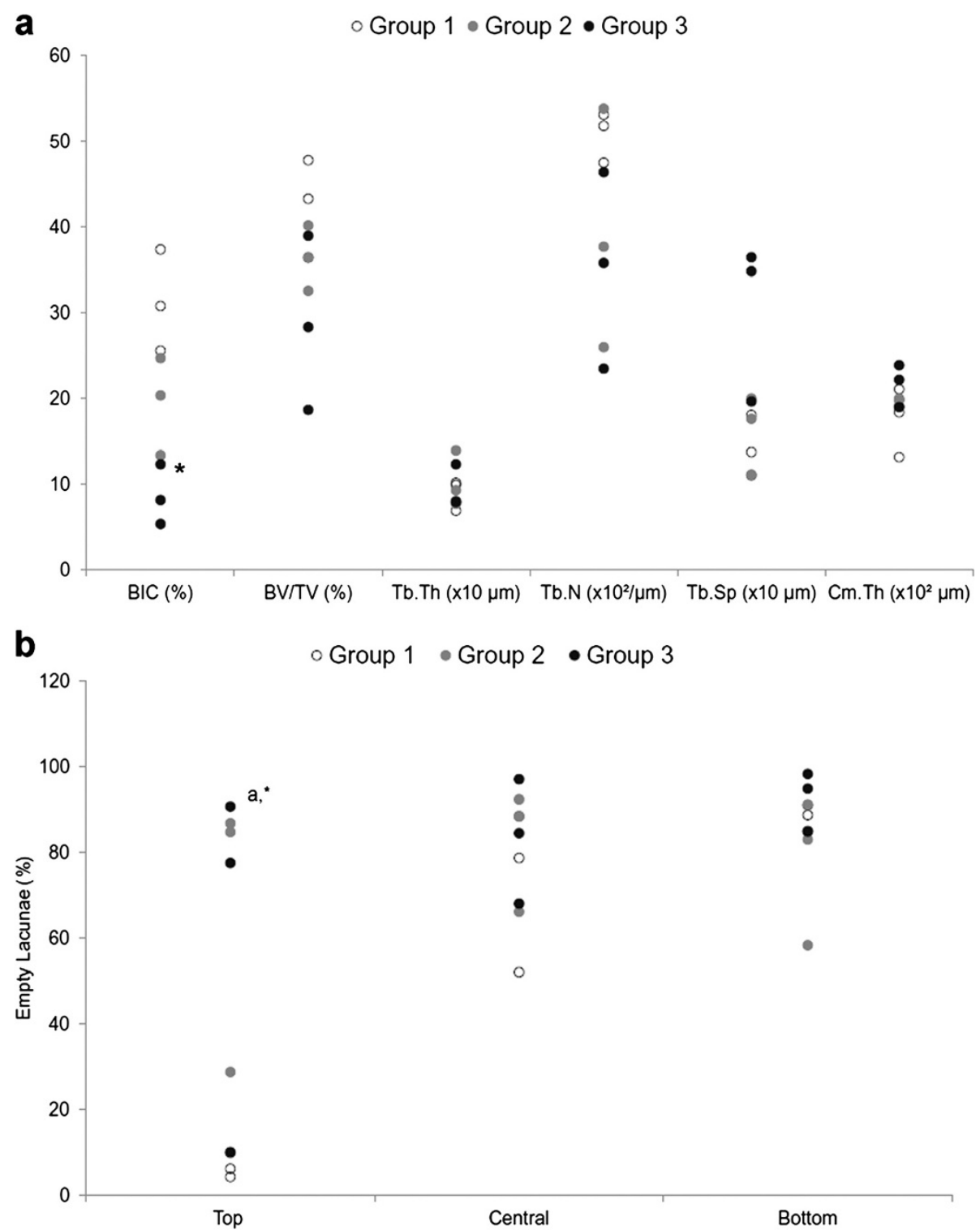

Figure 3 Dot plot of (a) histomorphometric parameters and (b) percentage of empty osteocyte lacunae in the different ROls (top, central, bottom) for each Group. Mann-Whitney test: (a) Group 3 versus Group $1\left(^{*}, p<0.05\right)$; (b) ${ }^{\text {a }}$, Top region versus central and bottom regions $(p<0.05)$. 


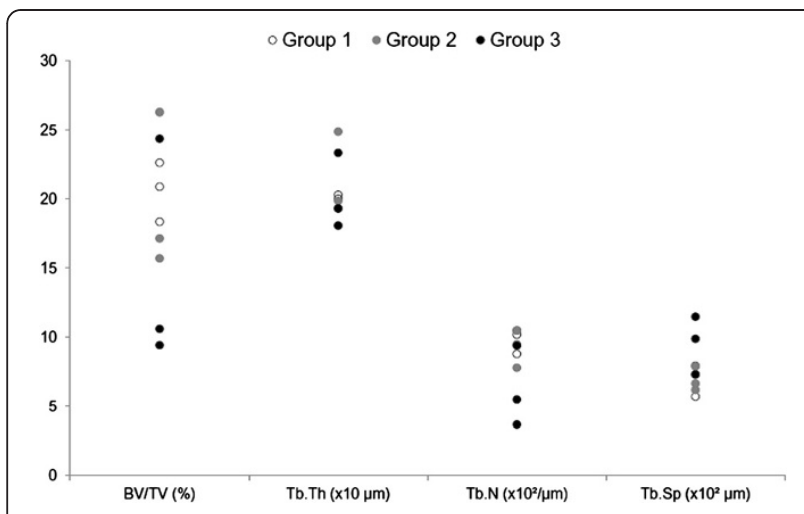

Figure 4 Dot plot of microtomographic analysis for each Group.

Group 3 showed that BV/TV and Tb.N decreased by about $28 \%$ and $37 \%$, respectively, whereas Tb.Sp increased by about $37 \%$, but the differences did not reach statistical significance. Microtomographic data obtained from each separate ROI (top, middle and bottom) are reported in Figure 6a-d. Statistically significant differences between Groups in the top ROI were found for BV/TV $(p=0.025)$, Tb.N $(p=0.025)$ and Tb.Sp $(p=0.028)$. In the top ROI, the Mann-Whitney test showed that Group 2 and Group 3 presented significantly lower BV/TV (Group 2: 61\%, $p=$ 0.05; Group 3: $41 \%, p=0.05$ ) and Tb.N (Group 2: 53\%, $p=0.05$; Group 3: 40\%, $p=0.05$ ), and higher Tb.Sp (Group 3: $71 \%, p=0.05$ ) when compared to those of Group 1.

\section{Discussion}

The main goal of this study was to evaluate the characteristics of bone quality and its microarchitecture in a series of femoral heads that failed at different times for different reasons by adopting an innovative and specific quantitative histomorphometry and $\mu \mathrm{CT}$ methodology. To do this nine failures were considered, which were split into groups depending on the failure time: 3 specimens failed at less than 6 months (Group 1), 3 failed between 6 months and 3 years (Group 2) and 3 failed at more than 3 years (Group 3) after HR surgery. In comparison with other studies, in this one the Groups were divided arbitrarily to highlight the possible presence of a phenomenon that progresses over time.

Histological evaluation showed the presence of focal areas of osteonecrosis with empty lacunae in the Group 1. In Group 2 and Group 3 partial osteonecrosis also was present; nevertheless, newly formed bone was visible on the surface of the necrotic bone trabeculae. These data were in agreement with those of Steffen et al. who showed that the necrotic changes were associated with appositional new bone formation and marrow fibrosis [14]. In fact, proliferating cells spread through the narrow spaces between the dead trabeculae, differentiate into osteoblast, and subsequently form appositional new bone on the surface of dead trabeculae. At the same time, they initiate osteoclastic resorption of necrotic bone. Osteoclastic resorption, modulated by cytokines released from osteoblast, is crucial for the balance of the repair processes. The bone may be markedly weakened if resorption occurs at the interface of the viable and dead bone, or if revascularization and new bone formation in necrotic areas is prevented by the formation of a fibrous scar [22]. Bone atrophy was observed at histological analyses only in Groups 2 and 3 and these results were confirmed by micro-CT (BV/TV, Tb.N, Tb.Sp) thus suggesting a possible role of mechanical factors (stress shielding). Metallosis, with infiltration and accumulation of metallic wear debris, was visible in Group 2 and 3. Therefore, as shown by other authors who studied the failure mechanism of $\mathrm{HR}$ prostheses by conventional radiography and qualitative histology, the present histological analyses confirmed that aseptic necrosis and bone rarefaction might play a crucial role in late failures of HR $[6,14,15,17,23-25]$.

Unlike previous studies, the present one took into consideration 3 groups of patients according to failure times (from 3 weeks to 8 years); quantitative measurements were performed with histomorphometry and $\mu \mathrm{CT}$ and 3 peri-implant bone regions at different distances from the HR dome (within $0.8 \mathrm{~cm}$ (top), from 0.8 to $1.6 \mathrm{~cm}$ (central) and from 1.6 to $2.4 \mathrm{~cm}$ (bottom)) were considered. This was possible through resin embedding of the femoral heads containing the prostheses, cutting along the coronal plane of the macro-sections and subsequent removal by pressure of the prosthesis that permitted the accurate evaluation of bone histology and microarchitecture with both 2D (histomorphometry) and $3 \mathrm{D}(\mu \mathrm{CT})$ techniques. A different bone architecture was highlighted within each group and, in particular,

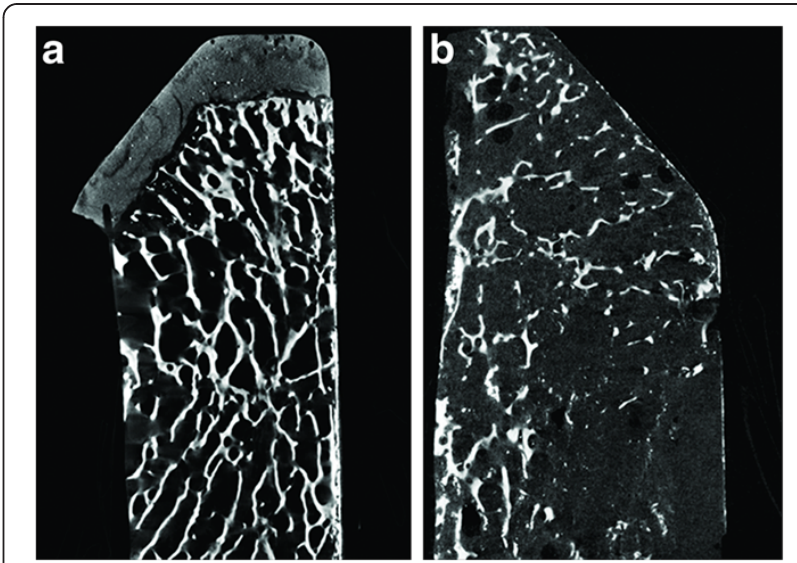

Figure 5 Microtomographic sections of HR failure after prostheses removal. a) Group 1 (5 months); b) Group 3 (7 years) showing an important bone rarefaction. 


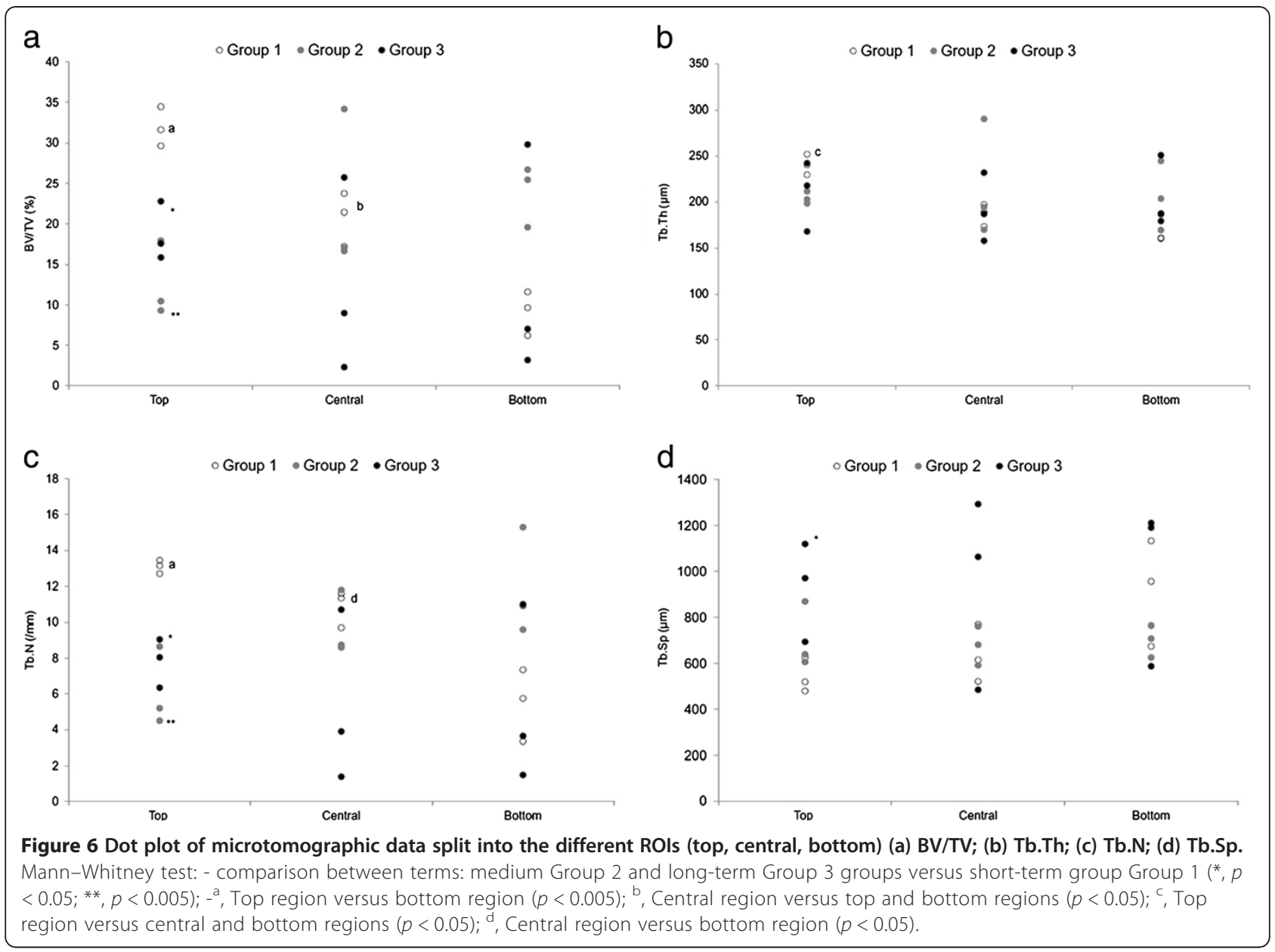

between the Group 1 and Group 3. Both 2D and 3D measurements showed that bone density decreases over time especially in Group 3 if compared with Group 2 and Group 1. 3D data of different ROIs (top, central, bottom) of both lateral and medial compartments showed a significant decrease in bone quality over time in the top ROI near the dome. This was confirmed by the significant differences in BV/TV, Tb.N and Tb.Sp between Group 1 versus Group 2 and Group 3 in the top ROI. This tendency was visible also in the lower ROIs but bone values did not reach statistical significance.

In the present study bone resorption was observed within the resurfaced femoral head and around the proximal part of the stem. Whereas bone remodeling is a feature of normal metabolism in healthy and osteoarthritic bone, the BHR may result in stress shielding with consequent resorption and narrowing of the femoral neck due to altered loading conditions. This stress shielding is probably due to the implant design with long-stems. In fact, Bidyut Pal and coworkers showed that bone resorption was considerably less for short-stem designs; the shortstem design having stem-bone contact not only led to a more physiological stress distribution but also to bone apposition in the superior side of the resurfaced head [26]. Moreover, 2D results showed significant differences also in the percentage of empty lacunae between Group 1 versus Group 2 and Group 3 in the top ROI. The proportion of empty lacunae gradually increased over the time after surgery.

The present results were in agreement with those of Steffen et al. who showed that samples from late fractures had a significantly higher proportion (84\%) of empty osteocyte lacunae within the trabecular bone compared with those of samples from fractures occurring within the first month (48\%) after HR [14]. Moreover, in the present study the higher mean percentage of empty lacunae in the central and bottom regions of Group 1 was probably due to a vascular injury. This controversial result might be explained by analyzing the surgical technique. During femoral head preparation the top region is always removed, thus eliminating the bone volume more subjected to osteonecrosis. Moreover, the residual blood supplied comes from the lateral femoral circumflex artery and a recent report [27] shows two 
more possible sources of blood supply to the femoral head. These two vessels were identified as the anterior nutrient artery of the femoral neck which origins from the lateral femoral circumflex artery and the inferior branch of the deep branch of the superior gluteal artery.

In the present series all the operations were performed through a posterior approach which is known to disrupt the medial circumflex artery; nevertheless, the failure rate due to bone necrosis was low. Similar findings were observed by Mcbride et al. who reported the same implant survival regardless of surgical approach [28]. By using a surgical approach that preserves the blood supply it might be possible to obtain an improved implant survival at longer follow up $[14,19,29]$.

The BIC measurement should not be considered as an index of osteointegration because the surgical procedure of HR insertion is not aimed at achieving primary fixation between the bone and the stem as for traditional arthroplasties. However, a progressive decrease of bone in contact around the stem was observed and the difference was significant between Group 1 and Group 3 patients. The decrease in BIC was probably due to the bone rarefaction, which involves the femoral head; it remains to be seen whether it might also be related to a progressive prosthesis loosening over time. In the present study histological and microtomographic analyses suggest that both processes, bone rarefaction and osteonecrosis, start from the bottom of the peri-implant bone and reach the top region adjacent to the HR dome in the Group 2 and 3. Osteonecrosis is expected to start from the top ROI which is far away from blood vessels and probably more influenced by the presence of cement but the findings in the present study showed the contrary. In fact, some sort of stress shielding due to its close relationship with the implant might be the true reason for this particular finding.

The current study has several limitations. First of all the small number of cases prevents any solid conclusions to be drawn about the real failure mechanisms of HR and the progression of femoral head damage. The inter-individual variability between patients and osseous changes should also be taken into account. Nevertheless, it was not the primary objective of this study to define the pathophysiology of HR prosthesis failure. To the present authors' knowledge, quantitative methodologies for measuring bone quality and its microarchitecture have never been used to study retrieved HR prostheses. In the present study the histomorphometric and microtomographic evaluations allowed bone microarchitecture alterations to be quantified.

\section{Conclusions}

The objective of the study was to examine the characteristics of bone quality and its microarchitecture in retrieved metal-on-metal HR by a specific quantitative histomorphometry and $\mu \mathrm{CT}$ method. The results showed that the morphometric parameters considered were crucial for a good understanding of the mechanical properties of HR and may be of significant and essential importance in the pathogenesis of HR failure particularly in the development of late fractures. Although there are several good reports on the survival rate of $\mathrm{HR}$ at midterm follow up, the biological changes of the femoral head underlying the implant over time should always be considered. It remains to be seen whether other late failures will occur. HR is still a good indication for young and active patients; nevertheless good bone quality remains the crucial element to support the implant at longer follow-up.

\section{Abbreviations}

HR: Resurfacing Arthroplasty; BHR: Birmingham Hip Resurfacing; $\mu C T$ : Microtomography; BV/TV: Bone Volume; Tb.N: Trabecular Number; Tb. Th: Trabecular Thickness; Tb.Sp: Trabecular Separation; Cm.Th: Cement Thickness.

\section{Competing interests}

The authors declare that they have no competing of interests.

\section{Authors' contributions}

FS provided input on the interpretation of the results and drafted the manuscript; MF participated in designing the study and helped edit the manuscript; AP carried out the microcomputer tomography analysis; MC participated in discussions of the project and revised it critically for important intellectual content; NNA provided input on the interpretation of the results; GG participated in designing the study and carried out the statistical analysis; DL provided input on the interpretation of the results; SG designed the study, provided input on the interpretation of the results and helped to draft the manuscript. All authors read and approved the final manuscript.

\section{Acknowledgements}

This work was supported by Rete Nazionale di Ricerca TISSUENET ( $\mathrm{n}$. RBPR05RSM2). None of the authors has professional and financial affiliations that may be perceived to have biased the presentation.

\section{Author details}

'Laboratory of Biocompatibility, Innovative Technologies and Advanced Therapies, Rizzoli Research Innovation Technology, Rizzoli Orthopaedic Institute, Via di Barbiano 1/10, 40136, Bologna, Italy. ${ }^{2}$ Laboratory of Preclinical and Surgical Studies, Rizzoli Orthopaedic Institute, Bologna, Italy. ${ }^{3} \|$ Orthopaedic and Traumatology Clinic, Rizzoli Orthopaedic Institute, Bologna, Italy.

Received: 11 April 2012 Accepted: 23 January 2013

Published: 30 January 2013

\section{References}

1. McMinn D, Treacy R, Lin K, Pynsent P: Metal on metal surface replacement of the hip: experience of the McMinn prosthesis. Clin Orthop 1996, 329:89-98.

2. van Gerwen M, Shaerf DA, Veen RM: Hip resurfacing arthroplasty. Acta Orthop 2010, 81(6):680-3.

3. McMinn DJ, Daniel J, Ziaee H, Pradhan C: Indications and results of hip resurfacing. Int Orthop 2011, 35(2):231-7.

4. Spierings PT: Hip resurfacing: expectations and limitations. Acta Orthop 2008, 79(6):727-30.

5. Shimmin AJ, Back D: Femoral neck fractures following Birmingham hip resurfacing: a national review of 50 cases. J Bone Joint Surg Br 2005, 87-B:463-474 
6. Zustin J, Krause M, Breer S, Hahn M, von Domarus C, Rüther W, Sauter G, Morlock MM, Amling M: Morphologic analysis of periprosthetic fractures after hip resurfacing arthroplasty. J Bone Joint Surg Am 2010, 92(2):404-10.

7. Prosser GH, Yates PJ, Wood DJ, Graves SE, de Steiger RN, Miller LN: Outcome of primary resurfacing hip replacement: evaluation of risk factors for early revision. Acta Orthop 2010, 81(1):66-71.

8. Daniel J, Pynsent PB, McMinn DJ: Metal-on-metal versus polyethylene in hip arthroplasty: a randomized clinical trial. Clin Orthop Relat Res 2004, 422:271.

9. Ullmark G, Sundgren K, Milbrink J, Nilsson O, Sörensen J: Osteonecrosis following resurfacing arthroplasty. Acta Orthop 2009, 80(6):670-4

10. Amstutz HC, Campbell PA, Le Duff MJ: Fracture of the neck of the femur after surface arthroplasty of the hip. J Bone Joint Surg Am 2004, 86:1874-7.

11. Glyn-Jones S, Pandit H, Kwon YM, Doll H, Gill HS, Murray DW: Risk factors for infammatory pseudotumour formation following hip resurfacing. J Bone Joint Surg Br 2009, 91(12):1566-1574.

12. Campbell $P$, Shimmin A, Walter L, Solomon M: Metal sensitivity as a cause of groin pain in metal-on-metal hip resurfacing. J Arthroplasty 2008, 23(7):1080-5.

13. Daniel J, Ziaee H, Kamali A, Pradhan C, Band T, McMinn DJ: Ten-year results of a double-heat-treated metal-on-metal hip resurfacing. J Bone Joint Surg Br 2010, 92(1):20-7.

14. Carrothers AD, Gilbert RE, Jaiswal A, Richardson JB: Birmingham hip resurfacing: the prevalence of failure. J Bone Joint Surg Br 2010, 92(10):1344-50.

15. Amstutz HC, Beaulé PE, Dorey FJ, Le Duff MJ, Campbell PA, Gruen TA: Metal-on-metal hybrid surface arthroplasty: two to six-year follow-up study. J Bone Joint Surg Am 2004, 86-A(1):28-39.

16. Treacy RB, McBryde CW, Shears E, Pynsent PB: Birmingham hip resurfacing: a minimum follow-up of ten years. J Bone Joint Surg $\mathrm{Br}$ 2011, 93(1):27-33.

17. Ollivere B, Darrah C, Barker T, Nolan J, Porteous MJ: Early clinical failure of the Birmingham metal-on-metal hip resurfacing is associated with metallosis and soft-tissue necrosis. J Bone Joint Surg Br 2009, 91(8): 1025-30.

18. Giannini S, Cadossi M, Chiarello E, Faldini C, Moroni A, Romagnoli M: Hip resurfacing arthroplasty: a series of 140 consecutive hips with a minimum five year follow-up. A clinical, radiological and histological analysis. Hip Int 2011, 21(1):52-58.

19. Hildebrand T, Rüegsegger P: A new method for the model-independent assessment of thickness in three-dimensional images. J Microscopy 1997, 185:67-75.

20. Martini L, Staffa G, Giavaresi G, Salamanna F, Parrilli A, Serchi E, Pressato D, Arcangeli E, Fini M: Long-term Results following a Cranial Hydroxyapatite Prosthesis implantation in a large Skull Defect Model. Plast Reconstr Surg 2012, 129(4):625-35.

21. Parfitt AM: Bone histomorphometry: standardization of nomenclature, symbols and units Summary of proposed system. Bone Miner 1988, 4(1):1-5.

22. Steffen RT, Athanasou NA, Gill HS, Murray DW: Avascular necrosis associated with fracture of the femoral neck after hip resurfacing: histological assessment of femoral bone from retrieval specimens. J Bone Joint Surg Br 2010, 92(6):787-93.

23. Mont MA, Jones LC, Einhorn TA, Hungerford DS, Reddi AH: Osteonecrosis of the femoral head. Potential treatment with growth and differentiation factors. Clin Orthop Relat Res 1998, 355(Suppl):314-335.

24. Morlock MM, Bishop N, Rüther W, Delling G, Hahn M: Biomechanical, morphological, and histological analysis of early failures in hip resurfacing arthroplasty. Proc Inst Mech Eng H 2006, 220(2):333-44.

25. Lazarinis S, Milbrink J, Hailer NP: Avascular necrosis and subsequent femoral neck fracture 3.5 years after hip resurfacing: a highly unusual late complication in the absence of risk factors--a case report. Acta Orthop 2008, 79(6):763-768.

26. Pal B, Gupta S, New AM: Influence of the change in stem length on the load transfer and bone remodelling for a cemented resurfaced femur. J Biomech 2010, 43(15):2908-14.
27. Zlotorowicz M, Czubak J, Kozinski P, Boguslawska-Walecka R: Imaging the vascularisation of the femoral head by $\mathrm{CT}$ angiography. J Bone Joint Surg Br 2012, 94(9):1176-9.

28. McBryde CW, Revell MP, Thomas AM, Treacy RB, Pynsent PB: The influence of surgical approach on outcome in Birmingham hip resurfacing. Clin Orthop Relat Res 2008, 466(4):920-6.

29. Ganz R, Gill TJ, Gautier E, Ganz K, Krügel N, Berlemann U: Surgical dislocation of the adult hip a technique with full access to the femoral head and acetabulum without the risk of avascular necrosis. J Bone Joint Surg Br 2001, 83(8):1119-24.

doi:10.1186/1471-2474-14-47

Cite this article as: Salamanna et al:: Histological, histomorphometric and microtomographic analyses of retrieval hip resurfacing arthroplasty failed at different times. BMC Musculoskeletal Disorders 2013 14:47.

\section{Submit your next manuscript to BioMed Central and take full advantage of:}

- Convenient online submission

- Thorough peer review

- No space constraints or color figure charges

- Immediate publication on acceptance

- Inclusion in PubMed, CAS, Scopus and Google Scholar

- Research which is freely available for redistribution 